\title{
E2 enzymes: more than just middle men
}

\author{
Mikaela D Stewart ${ }^{1, *}$, Tobias Ritterhoff ${ }^{1, *}$, Rachel E Klevit ${ }^{1}$, Peter S Brzovic ${ }^{1}$ \\ ${ }^{I}$ Department of Biochemistry, University of Washington, Seattle, WA 98102 USA
}

Ubiquitin-conjugating enzymes (E2s) are the central players in the trio of enzymes responsible for the attachment of ubiquitin (Ub) to cellular proteins. Humans have $40 \mathrm{E2s}$ that are involved in the transfer of Ub or Ub-like (Ubl) proteins (e.g., SUMO and NEDD8). Although the majority of E2s are only twice the size of Ub, this remarkable family of enzymes performs a variety of functional roles. In this review, we summarize common functional and structural features that define unifying themes among E2s and highlight emerging concepts in the mechanism and regulation of E2s.

Keywords: E2; ubiquitin-conjugating enzymes; HECT; RING

Cell Research (2016) 26:423-440. doi:10.1038/cr.2016.35; published online 22 March 2016

\section{Introduction}

Nearly every paper on protein ubiquitylation describes the mandatory sequence of enzyme activities required for target modification. The process starts with activation of the ubiquitin (Ub) C-terminus by a ubiquitin-activating enzyme (E1) in an ATP-dependent reaction to generate a thioester-linked E1 Ub conjugate. The activated $\mathrm{Ub}$ is then handed over to a ubiquitin-conjugating enzyme (E2) via a transthiolation reaction. Finally, a ubiquitin ligase (E3), which can bind both a substrate and an E2 Ub conjugate, mediates the transfer of $\mathrm{Ub}$ most commonly onto the $\varepsilon$-amino group of a lysine in the target protein, forming an isopeptide bond. On the basis of their mechanistic strategies, E3s have been classified into three families (i.e., RING-, HECT-, and RING-between-RINGS (RBR)type E3s) and have been intensely studied. However, the central player in this enzymatic choreography, the E2, is often viewed as simply a carrier of Ub. Although the majority of E2s are only twice the size of Ub, this remarkable family of enzymes performs a variety of functional roles. With only one simple active site, E2s primarily engage in two types of reactions as follows: (1) transthiolation (transfer from a thioester to a thiol group) and (2) aminolysis (transfer from a thioester to an amino group), although additional types of reactivity have been report-

*These two authors contributed equally to this work.

Correspondence: Rachel E Klevit

E-mail:klevit@u.washington.edu ed (Figure 1B). All E2s interact with an E1 enzyme and one or more E3s. In addition, E2s may directly engage a target protein and so play a role in the determination of where and how a target is modified by Ub [1]. Finally, some E2s also function outside the traditional Ub transfer pathways to regulate the activity of other enzymes [2,3].

Humans have $\sim 40$ E2s that are involved in the transfer of Ub or Ub-like (Ubl) proteins (e.g., SUMO, NEDD8, etc). All E2s contain a core catalytic domain of roughly 150 amino acids, termed the UBC domain (Figure 1A). This domain adopts an $\alpha / \beta$-fold typically with four $\alpha$-helices and a four stranded $\beta$-sheet. Important loop regions form part of the E3-binding site (see below) and the E2 active site. Structures of over 32 human E2 proteins (fulllength or UBC domain) have been solved and the topologies of most are remarkably consistent with this canonical fold. In a few instances, such as Ube2R1 (Cdc34) or Ube2G2 (Figure 1A), the UBC domain is embellished with functionally important insertions. Although most E2s encompass only a single structural UBC domain, many have short $\mathrm{N}$ - and/or C-terminal extensions that can impart important E2-specific functionality (Table 1). Although extensions are often intrinsically disordered, a few adopt secondary structure that then contacts the UBC domain [4]. Furthermore, a few E2s have an additional structured domain linked to their UBC domain (e.g., Ube2K) or are part of large multi-domain proteins (e.g., Ube2O or BIRC6). Thus, E2s have a common fold that has been adapted for specific systems. Rather than discussing what is known about each E2, this review endeavors to identify common functional and structural 


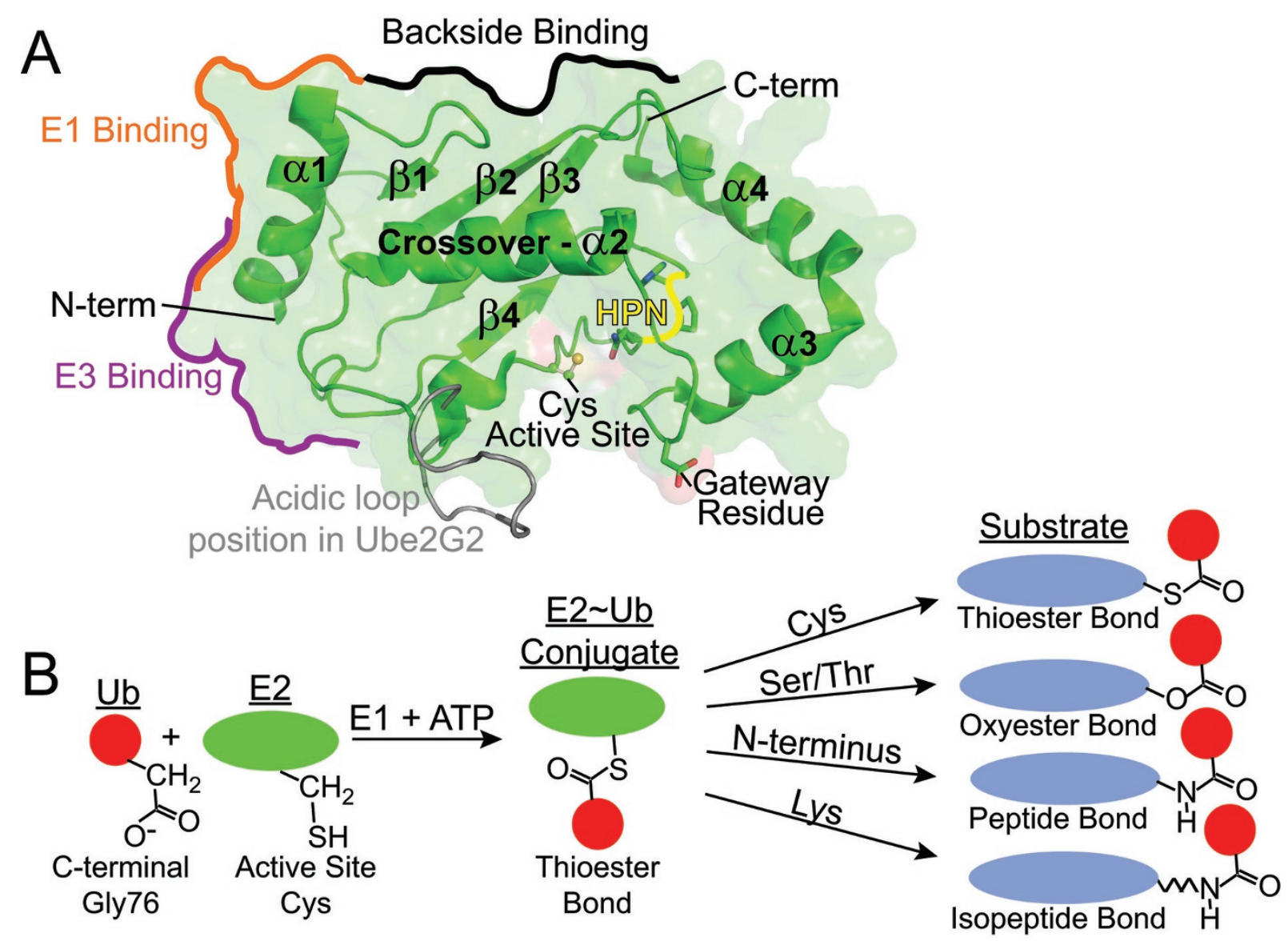

Figure 1 Overview of E2 structure and chemistry. (A) Important structural features and common binding surfaces described in the text are labeled on a representative UBC domain (Ube2D3; PDBID: 2FUH) shown in green. The position of the acidic loop in Ube2G2 (PDBID: 2CYX) when the UBC domain is aligned with Ube2D3 is shown in gray. (B) The C-terminal carboxylate of $\mathrm{Ub}$ is conjugated to the E2 active site cysteine in an E1-catalyzed, ATP-driven reaction. The E2 Ub conjugate reacts with the side chain of a Cys, Ser, Thr, Lys, or N-terminus on a substrate to form the diverse ubiquitin linkages as shown.

features that define unifying themes. We also highlight emerging concepts from the recent literature to help guide future studies.

\section{E2 reactivity}

In a cell, E2s exist mainly as E2 Ub conjugates and are therefore poised to react [5]. However, E2 Ub conjugates have low rates of $\mathrm{Ub}$ transfer in the absence of an E3 ligase, presumably to avoid energy-costly cycles of conjugation and off-target ubiquitylation. A long-standing question in the field is "What keeps the reactivity of an E2 Ub low?" or put another way "How is reactivity stimulated by E3s?". Studies over the past several years provide insights into both of these fundamental questions. A full understanding requires characterization of $\mathrm{E} 2$ reactivity per se and of how an E3 alters it.
Intrinsic reactivity of $E 2 s$

The ability of E2 $\mathrm{Ub}$ conjugates to transfer Ub can be assessed by gauging their intrinsic reactivity towards small-molecule nucleophiles such as free lysine (aminolysis) or cysteine (transthiolation) [6].This assay strips away complications inherent in other standard in vitro assays such as the highly popular auto-ubiquitylation assays or assays that use "universal" substrates such as histone 2A [6]. Use of a single (small) substrate that carries the nucleophilic functional group of interest allows the reactivity of different E2 Ub conjugates to be compared directly in the presence and absence of an E3. Presumably, all E2s can transfer Ub via transthiolation, a requirement for activation by an $\mathrm{E} 1$. In the most common example of a transthiolation reaction, an E2 transfers its thioester-linked $\mathrm{Ub}$ to the active-site cysteine residue of a HECT-type E3 ligase (for a review of E3 mechanisms, 


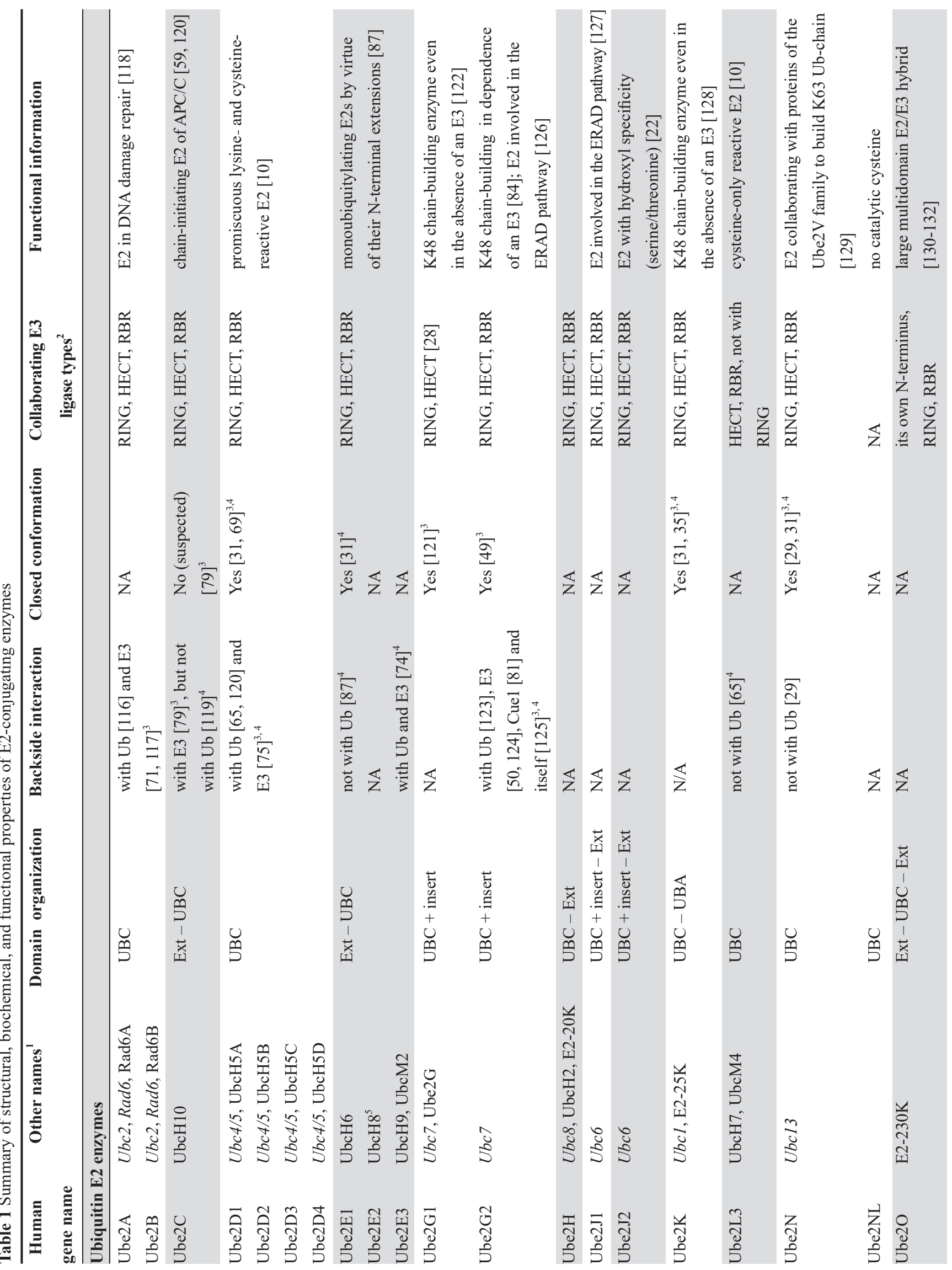



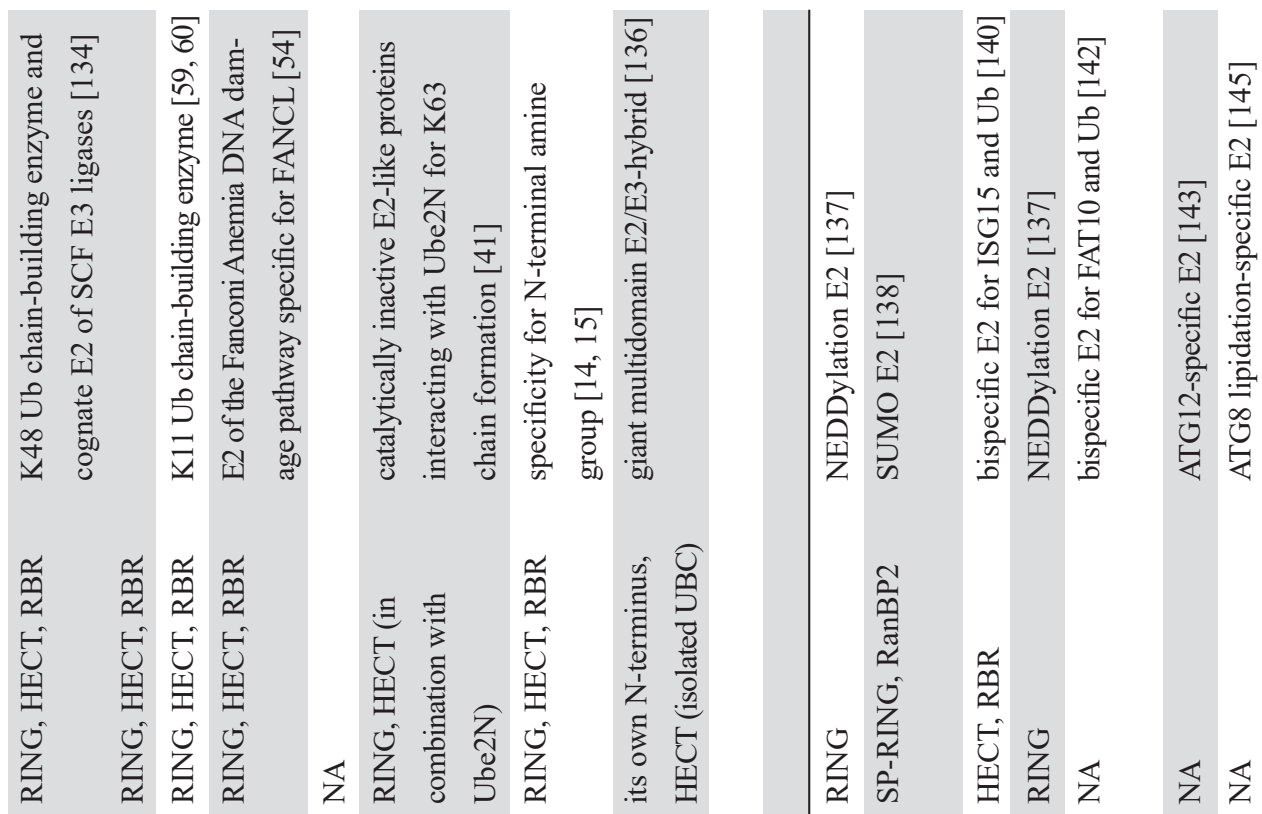

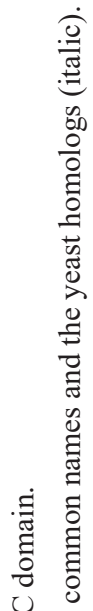

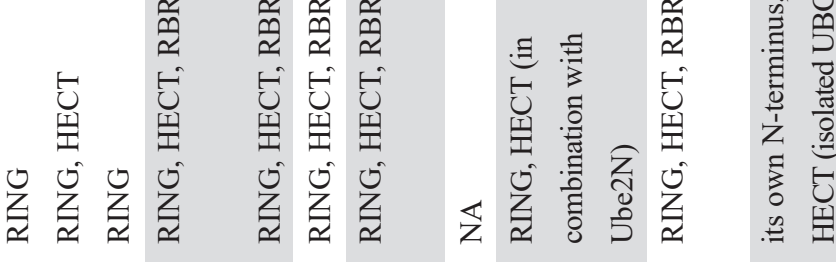

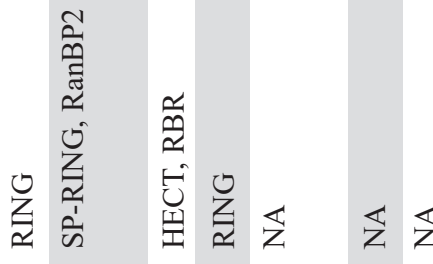

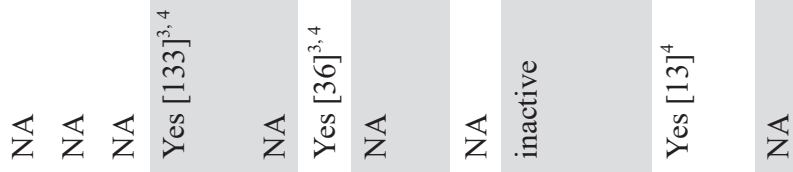

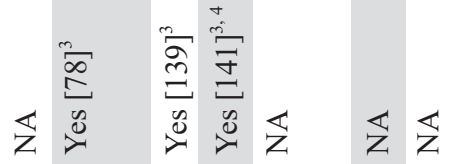

离

㻤

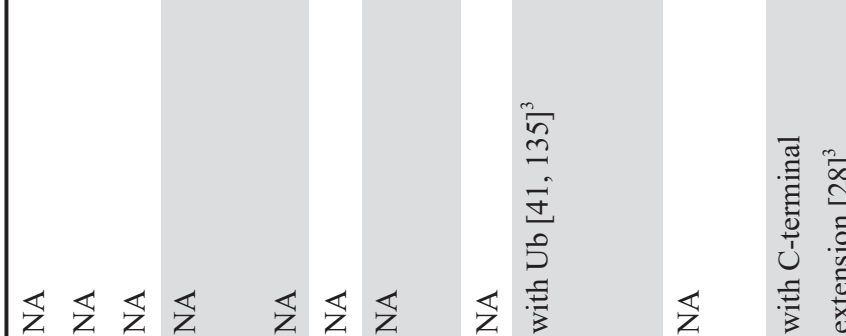

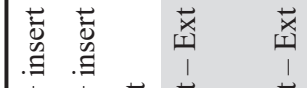

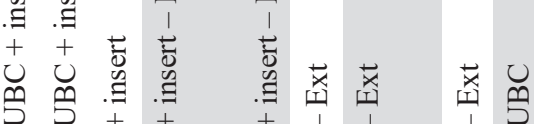

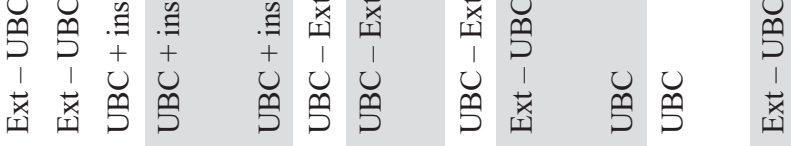

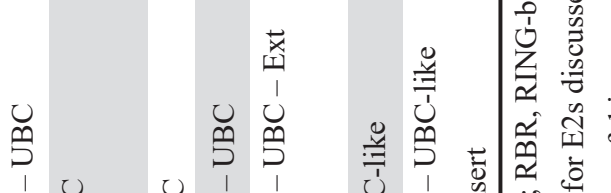

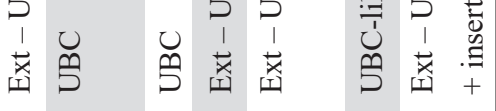

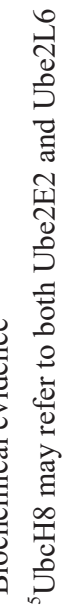

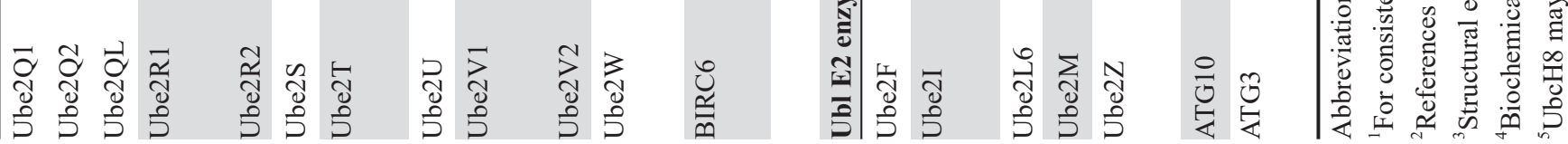


see Metzger et al. [7]). There is, however, one reported example of an E2 transferring $\mathrm{Ub}$ to a cysteine residue on a substrate. The yeast peroxisome E2 Pex2 modifies the peroxisomal receptors Pex $5 p$ and Pex $10 p$ on conserved and functionally essential cysteine residues $[8,9]$. Although a complete assessment of all human E2s has not yet been reported, most Ub E2s examined to date also show some propensity to transfer $\mathrm{Ub}$ to free lysine, consistent with their ability to work as E2s with the largest class of E3 ligases, the RING-type E3 (see discussion below).

The intrinsic reactivity assay has uncovered unexpected features of some E2s that have changed the way we think about E2s and E3s. Notably, Ube2L3 (UbcH7), the E2 used in many early structural studies with RINGtype E3s, is not reactive towards lysine and only exhibits reactivity towards cysteine [10]. The implication is that Ube2L3, although it binds to many RING domains, is only functional as an E2 with HECT-type E3s. This observation and its implication were key in redefining an entire class of E3s, the RBRs that were known to function with Ube2L3. RBRs, such as Parkin and HHARI, have RING domains, but also contain a conserved cysteine residue that forms an obligatory E3 Ub intermediate. Thus, RBRs are functional hybrids that exploit elements found in both RING and HECT E3s [10].

Another example of unusual intrinsic E2 reactivity is Ube2W. Although known to work with E3s typically associated with product lysine ubiquitylation such as BRCA1/BARD1 and FANCL [11, 12], Ube2W exhibits no intrinsic activity towards free lysine [13]. Instead, Ube $2 \mathrm{~W}$ attaches $\mathrm{Ub}$ to the $\mathrm{N}$-terminal $\alpha$-amino group of proteins to form a Ub-fusion protein product $[14,15]$. While still an aminolysis reaction and therefore not fundamentally different from the reaction with lysine, intrinsic reactivity assays revealed that Ube $2 \mathrm{~W}$ can transfer $\mathrm{Ub}$ to the $\alpha$-amino group of small lysine-less peptides but not to free lysine, whereas Ube2D3, for example, can transfer $\mathrm{Ub}$ to lysine but not to the $\alpha$-amino group [13]. This feature distinguishes Ube $2 \mathrm{~W}$ as fundamentally different in its reactivity (and therefore, its substrates) from all other characterized E2s. Along with its unique reactivity profile, Ube $2 \mathrm{~W}$ has an unusual UBC domain, in which its C-terminal region is disordered and dynamic but hovers near the active site $[13,16]$. Ube $2 \mathrm{~W}$ recognizes and modifies disordered N-termini independently of substrate sequence through interactions between its own disordered C-terminal region and the substrate backbone [13]. The requirement of a disordered $\mathrm{N}$-terminus on its substrate explains the strict monoubiquitylating activity of Ube $2 \mathrm{~W}$, as the N-terminus of Ub is highly structured and is therefore not a good substrate for Ube $2 \mathrm{~W}$. There are recent reports that the preference for $\mathrm{N}$-terminal modification by Ube $2 \mathrm{~W}$ may not be absolute, as the retroviral restriction RING E3 TRIM5 $\alpha$ is monoubiquitylated by Ube2W despite being acetylated on its N-terminus [17]. This suggests that Ube $2 \mathrm{~W}$ may also facilitate isopeptide bond formation, possibly if an N-terminus is blocked. Nevertheless, the preference of Ube2W for disordered $\mathrm{N}$-termini gives it a (so far) unique target selection mechanism for a primary modification event that can subsequently be exploited by other E2 enzymes to form $\mathrm{Ub}$ chains (discussed below).

In an intriguing twist, a non-protein primary amine is targeted by ATG3, an E2 that plays a role in the expansion of autophagosomal membranes. ATG3 conjugates Ubl proteins of the LC3/ATG8 family to phosphatidylethanolamine in the outer phagosome membrane [18, 19]. The aminolysis reaction catalyzed by ATG3 results in the formation of an amide bond, which covalently links a Ubl protein to a phospholipid to anchor it to the membrane.

In addition to the documented reactivity of E2s with thiol and amino groups, other chemical reactivities have been reported for some (unusual) E2s [20]. Ube2J2 has been reported to attach $\mathrm{Ub}$ to the major histocompatibility complex via hydroxyl groups (serine/threonine) in collaboration with a viral RING E3 ligase, murine K3 (mK3) [21, 22]. Consistent with this proposal, Ubc6, a yeast homolog of Ube2J2, is essential for degradation of a lysine-less substrate [23]. Ube2J2 ubiquitylation products are sensitive to treatment with strong base, which hydrolyzes oxyesters but not amide bonds. Normally, the hydroxyl groups on serine, threonine, and tyrosine are not particularly nucleophilic, implying that the active site of Ube2J2 may possess additional catalytic residue(s) beyond those in most E2s. To date, the intrinsic ability of the Ube2J2 Ub conjugate to react with serine or threonine has not been demonstrated, so neither the structural nor chemical determinants for hydroxyl attachment of $\mathrm{Ub}$ have been identified. In summary, the growing examples of non-canonical Ub transfer by E2s highlight the need to explicitly test for ubiquitylation on non-lysine residues in substrates. To date, there are no examples where an E3 alters the chemical reactivity profile of an E2, so the intrinsic reactivity of a given E2 will likely be predictive of the nature of its products.

\section{Enhancement of E2 reactivity by E3 ligases}

There are two main classes of E3s with which E2s function: RING-type and HECT-type E3s. Together, these E3s comprise $\sim 700$ proteins in the human genome. A third smaller class, the RBRs, combines elements of both RING and HECT E3s. E3s from pathogenic bacteria 
that operate in eukaryotic cells and use the host ubiquitylation machinery (including E2s) may constitute other classes. Although the E3 classes are topologically distinct, some E2s have been shown to cross class boundaries and function with multiple types of E3s (Table 1). Much of the early structural work aimed at understanding E2 function focused on how they recognize E3s. Most E2/E3 complexes are characterized by moderate to weak affinity and cannot be co-purified. Nevertheless, NMR and crystallography studies helped define a canonical E3-binding surface on the UBC domain formed by residues in helix 1 , and loops 4 and $7[24,25]$. This surface is involved in binding to both RING and HECT domains and also overlaps with the region recognized by E1 enzymes (Figure 1A) [26, 27]. That this one region is responsible for multiple protein interactions may explain why many Ub-specific E2s exhibit similar electrostatic properties in this region of the UBC domain [28].

E3/E2 complexes, however, represent the complex that exists after the Ub transfer reaction has occurred. Substantial progress has been made in understanding the properties of E2 Ub conjugates and the structural changes that occur when they interact with an E3 and are poised for $\mathrm{Ub}$ transfer. The $\mathrm{C}$-terminal residues of $\mathrm{Ub}$ (amino acids 72-76) are highly flexible and allow a $\mathrm{Ub}$ molecule that is covalently tethered to an E2 active site to swing by its tail, sampling an ensemble of conformations relative to the E2 domain. Solution studies of Ube2D3 Ub and Ube $2 \mathrm{~N} \sim \mathrm{Ub}$ conjugates show an array of orientations that involve little or no contact between the E2 and ubiquitin ("open states") and some conformations ("closed states") that involve contacts between the Ub hydrophobic patch centered on Ub I44 and residues in the E2 crossover helix (Figure 2). Remarkably, although Ube2D3 $\mathrm{Ub}$ and Ube $2 \mathrm{~N} \sim \mathrm{Ub}$ conjugates are highly dynamic, the ensembles of conformations adopted by them are different in terms of the relative fraction of "closed" versus "open" states [29].

RING-type E3s, which include RINGs and the structurally related U-box proteins, make up the vast majority of E3 ligases (for a recent review, see Metzger et al. [7]). Unlike HECT and RBR E3 ligases that form E3 Ub intermediates during $\mathrm{Ub}$ transfer, RING E3s do not participate chemically in Ub transfer. Instead, RING E3s bind substrate and an E2 Ub conjugate to facilitate $\mathrm{Ub}$ transfer directly from the E2 active site to a substrate. In this respect, a RING-type E3 functions as a required protein co-factor for E2 Ub conjugates. Although E2 Ub conjugates may bind some E3s tighter than the free E2 counterpart, NMR studies show that RING E3/E2 Ub complexes are still dynamic [30]. Nonetheless, interaction with RING E3s dramatically enhances the intrinsic reactivity of many, although not all, E2 Ub conjugates
A

Open States
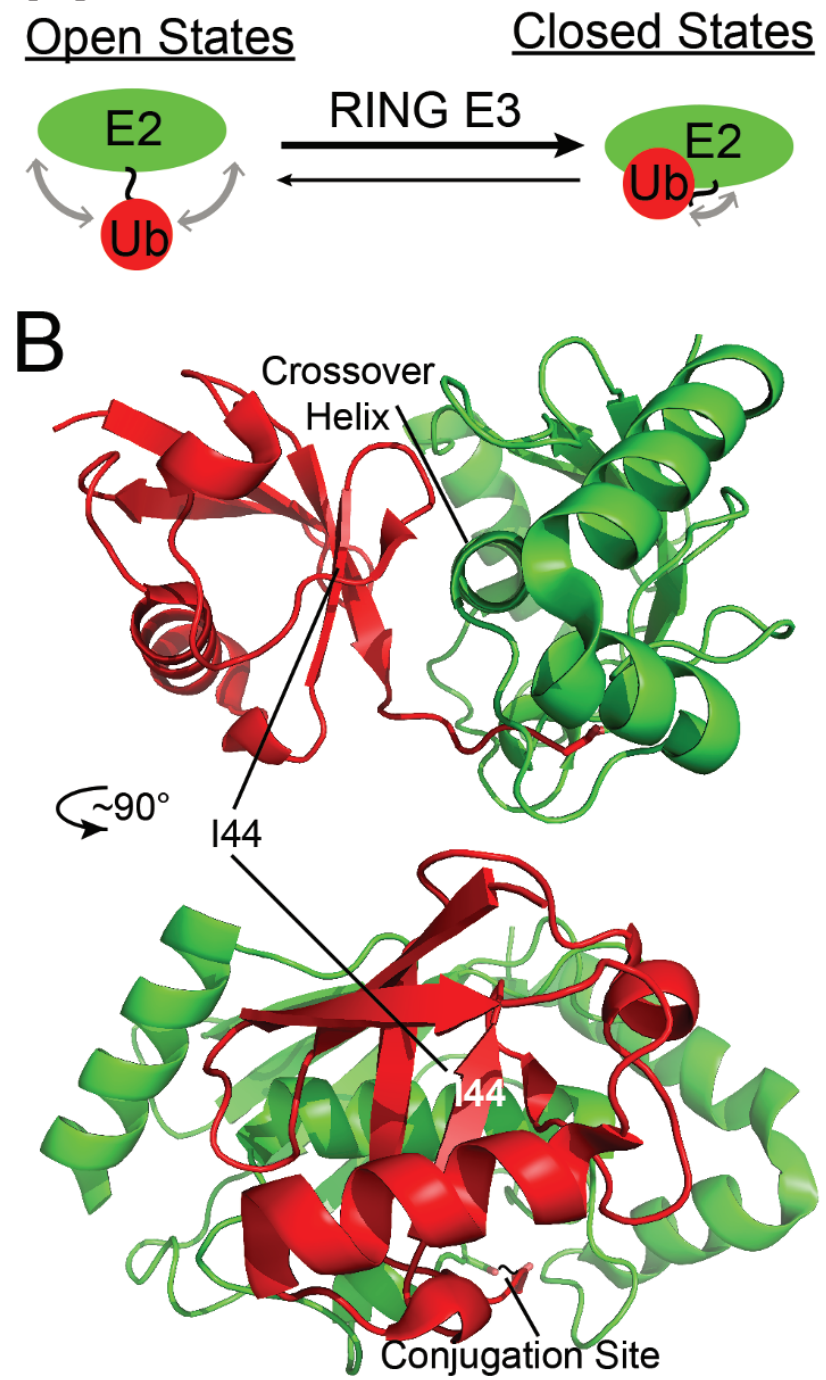

Figure 2 Ubiquitin positioning in the E2 Ub conjugate. (A) A cartoon depiction of the dynamic "open states" in which Ub samples a range of various positions relative to the $E 2$, but shifts towards population of more "closed states" upon binding of a RING-type E3. (B) Crystal structure of an E2 Ub oxyester conjugate (Ube2D2) in a closed state showing the interface formed between the E2 (green) crossover helix and the 144 surface of Ub (red) upon binding a RING E3 (BIRC7 (not shown); PDBID: $4 A \cup Q$ ). The two representations are the same structure rotated $\sim 90^{\circ}$ about the vertical axis. The E2 is in the same orientation as in Figure 1 in the bottom representation.

towards aminolysis. As a paradigm example, members of the Ube2D family of E2s react with lysine slowly in the absence of an E3 but rapidly in the presence of a RING domain [10]. Solution studies found that RING interactions promote E2 Ub closed states [31]. Snapshots of closed conformations have been captured in recent crystal structures [32-34]. 
Surprisingly, the E2/E3 interaction critical for promoting the closed E2 $\sim \mathrm{Ub}$ conformation occurs over $15 \AA$ away from the E2 active site. E3/E2 Ub structures reveal that a conserved RING residue donates a hydrogen bond to an E2 backbone carbonyl in loop 7 and one or more backbone groups in the tail of $\mathrm{Ub}[32,33]$. Structurally, the conserved RING residue (usually an arginine, lysine, or asparagine) appears to position the Ub C-terminus for nucleophilic attack. Substitution of the RING residue with a non-hydrogen-bonding residue abrogates activation of the E2 Ub by RING E3s, so it was dubbed the "allosteric linchpin" residue [31]. Furthermore, destabilization of closed E2 Ub states by mutations in the E2/ $\mathrm{Ub}$ interface also decreases the ability of RING E3s to activate the E2 $\mathrm{Ub}$ for $\mathrm{Ub}$ transfer. Taken together, these results have led to the notion that closed E2 $\sim \mathrm{Ub}$ states are activated states for aminolysis. Unfortunately, use of stable mimics of the thioester linkage (e.g., oxyesteror isopeptide-linked E2 Ub species), required to obtain crystals of E2 Ub conjugates, yields structures that do not contain the atoms that undergo the relevant chemistry. So the underlying activation mechanism remains unclear. Studies on a growing number of E2s and E3s confirm that promotion of E2 Ub closed states by RINGs is a common mechanistic strategy shared among many, although not all, E2/RING E3 pairs (Table 1). Finally, consistent with the notion that closed states are more reactive in aminolysis, the intrinsic lysine reactivity of an E2 Ub conjugate in the absence of a RING E3 appears to correlate with its natural tendency to populate closed states, although more examples are required to confirm this prediction $[29,35,36]$.

As more E2s undergo thorough investigation, exceptions and/or variations to the general mechanism are emerging. An intriguing example is Ube2S, the dedicated $\mathrm{E} 2$ for the multi-subunit APC/C E3 that regulates cell cycle progression. On its own, Ube $2 \mathrm{~S} \sim \mathrm{Ub}$ populates closed states to a considerable extent and can catalyze formation of free polyUb chains in the absence of an E3 [36]. Notably, mutations designed to disrupt canonical E2/ RING interactions that would involve the APC/C RING domain subunit (Apc11) do not affect activity [37, 38]. On the other hand, two non-RING subunits, Apc2 and Apc4, contribute to Ube2S activation in a poorly understood mechanism that may involve the $\mathrm{C}$-terminal helix of the Ube2S UBC domain [37]. It is tempting to speculate that the novel mechanism used by $\mathrm{APC} / \mathrm{C}$ to activate its dedicated E2 ensures that APC/C substrates are not ubiquitylated at the wrong place or time. Undoubtedly, new variations of the general mechanism will continue to emerge in the future.

In contrast to the dependence of aminolysis on closed
E2 Ub conformations, E2 Ub conjugates readily undergo transthiolation reactions in the absence of E3s. This implies that E3s that use a HECT-type mechanism (i.e., progress via an E3 Ub conjugate intermediate) do not need to promote closed states. This prediction is supported by structures of E2 Ub bound to a HECT (NEDD4L) or RBR (HOIP) E3, which reveal a Ube2D2 Ub conjugate in an open conformation, poised for transthiolation to the E3 active-site cysteine [39, 40]. Consistent with the notion that aminolysis depends on closed E2 Ub states, intrinsic reactivity assays show that neither HECTs nor RBRs enhance the intrinsic lysine reactivity of Ube2D3 [31]. A corollary is that, by trapping open E2 Ub, HECTtype E3s position their active-site cysteine for transthiolation and inhibit serendipitous transfer to any nearby lysines. This prediction has been confirmed in the complex of a pathogenic bacterial effector, OspG, that traps Ube2D3 Ub in an open conformation similar to that observed in the NEDD4L complex [3]. In this high-affinity complex, Ube2D3 Ub is highly reactive towards free cysteine, but shows greatly reduced reactivity towards free lysine when compared with free Ube2D3 Ub.

\section{Product determination by E2s}

A major question is how residue specificity is achieved during ubiquitylation. For HECT-type enzymes that transfer $\mathrm{Ub}$ via an $\mathrm{E} 3 \sim \mathrm{Ub}$ intermediate prior to the ultimate transfer to substrate, specificity is enforced primarily by the E3. However, for RING-type E3s, which represent the vast majority of $\mathrm{E} 3 \mathrm{~s}$, and for situations that are E3-independent, the E2 plays a determining role. With this in mind, we coin the new old adage: "The last guy holding the activated $\mathrm{Ub} / \mathrm{Ubl}$ gets to determine the product". In this regard, it is useful to categorize E2s in terms of their general product proclivities: (1) E2s that transfer $\mathrm{Ub} / \mathrm{Ubl}$ onto a target protein residue (monoubiquitylating or priming E2s); (2) E2s that transfer $\mathrm{Ub} / \mathrm{Ubl}$ onto another Ub/Ubl (chain-building E2s); and (3) promiscuous E2s that can do either. The distinction between transferring $\mathrm{Ub}$ to a lysine on a target protein versus a lysine on $\mathrm{Ub}$ itself implies different determinants for specificity. While there are numerous examples of each type of E2, current understanding is most advanced for chain-building E2s, although progress is being made on all fronts.

It is easy to imagine that an E2 that catalyzes formation of a linkage-specific polyUb chain will have evolved determinants to recognize the specified lysine residue presented within its structural and/or sequence context. Whereas K63-specific Ube2N uses a tightly bound E2like subunit (either Ube2V1 or Ube2V2) to position the K63 side chain of the incoming (acceptor) Ub [41], 
other linkage-specific E2s are able to specify their product using only their $\mathrm{UBC}$ domains. Ube $2 \mathrm{~K}$ has a unique region near its active site that interacts with a tyrosine near K48 in the acceptor $\mathrm{Ub}$ to provide K48-linkage specificity. Ube2S uses acidic residues in the final UBC domain helix to interact with the acceptor $\mathrm{Ub}$ and orient $\mathrm{K} 11$ towards the $\mathrm{C}$-terminus of the donor $\mathrm{Ub}$ bound to its active site [36, 42, 43]. A family of K48-specific E2s that includes Ube2R1, Ube2R2, Ube2G1, Ube2G2, and yeast Ubc7 has a short $\sim 12$ amino acid insertion proximal to the E2 active site that determines specificity (Figure 1A). This short, highly flexible element appears to dictate product formation using a number of strategies (discussed below).

Ube2R1 and its yeast counterpart $\mathrm{Cdc} 34$ are dedicated E2s for the large multi-subunit SCF (Skp/Cullin/F-Box) $\mathrm{E} 3 \mathrm{~s}$ that target proteins to the proteasome for degradation. Both of these enzymes also have an acidic C-terminal extension that interacts with a "basic canyon" on the cullin subunit of an SCF complex, helping to position the E2 near the RING subunit while allowing for rapid association and turnover in chain building [44]. In these E2s, the aforementioned short insertion, termed the acidic loop, affects function in several ways. First, the loop of Cdc34 interacts directly with the SCF complex [44, 45]. Use of additional, special structural features for E3 binding could serve to limit the ability of other cellular E2s to bind and be activated by the SCF, possibly generating products other than the desired K48-linked polyUb chains on SCF substrates. Second, the acidic loop helps orient the donor $\mathrm{Ub}$ for attack by the incoming K48 on the acceptor $\mathrm{Ub}$. For the Cdc34 Ub conjugate, positioning the donor $\mathrm{Ub}$ appears to set the stage for binding of the acceptor $\mathrm{Ub}$, allowing recognition of $\mathrm{Ub}$ residues in a loop located between residues E51 and Y59 to properly position the acceptor K48 within the E2 active site [46]. Third, the acidic loop appears to play a role in lowering the $\mathrm{pK}_{\mathrm{a}}$ of an as yet unidentified ionizable group on the $\mathrm{E} 2$, Ub, or even possibly the incoming $\mathrm{K} 48$, which must be deprotonated to become nucleophilic [45]. Altogether, these interactions promote the efficiency and fidelity of K48-linked polyUb chain synthesis by Ube2R1 and its homologues [45, 47, 48]. In the case of Ube2G2, binding of a non-RING region ("G2BR") of its E3, gp78, to the backside of the UBC domain (see Figure 1A and discussed in more detail below) alters the acidic loop conformation, which is helical in the free E2 structure but is unwound in the G2BR-bound structure $[49,50]$. The unwinding generates a series of interactions among E2, $\mathrm{E} 3$, and $\mathrm{Ub}$ that help stabilize a closed E2 Ub conformation to increase aminolysis reactivity. Thus, similar to the "extra" E2/E3 interaction between the Cdc34 acidic loop and its RING E3, the requirement of an extra, allosteric interaction between Ube2G2 and gp78 ensures that the K48 chain-building E2 cannot work with any RING E3 it happens to contact.

Although our understanding of how product specificity is dictated is fairly detailed when Ub serves as the incoming source of lysine, the same question regarding non-Ub substrates remains a thorny one. There are likely several reasons for this. First is the sheer number of E2s that can transfer $\mathrm{Ub}$ in cells and the difficulty of defining relevant E2/E3/target ensembles. Second is the even larger number of potential substrates. Third is the confounding situation that only a tiny minority of all targets are modified on specific residues. This is likely because in the case of polyubiquitylation, it is the chain itself that serves as the recognition element for further actions, so exactly where on a target protein that chain is attached is of little importance. This provides a rationale for the observation that most of the targets in which a specific residue is modified have only a single $\mathrm{Ub}$ (or Ubl) attached and therefore represent a very different type of product and outcome.

By far, the best-characterized E2 that transfers a Ub or Ubl moiety directly to a target residue is the sole SUMO E2 Ube2I (Ubc9). In the absence of an E3, Ube2I can modify targets that contain a lysine in a $\psi-\mathrm{K}-\mathrm{X}-\mathrm{D} / \mathrm{E}$ consensus motif (with $\psi$ being a bulky hydrophobic and $\mathrm{X}$ being any amino acid), if the motif is present in a loop or unstructured region [51, 52]. The active site of Ube2I positions the incoming lysine in this sequence context optimally for the nucleophilic attack on the activated SUMO thioester bond $[51,53]$. Although the consensus sequence is not an absolute requirement for Ube2I-dependent SUMOylation, this example illustrates the intimate contact between an E2 and a target that occurs during direct transfer reactions. Much less is known about the Ub E2s that target specific lysine residues and how this is achieved. For instance, Ube2T monoubiquitylates its substrate FANCD2 on a specific lysine with its RING E3 FANCL in the Fanconi Anemia DNA repair pathway [54]. The yeast E2 Rad6, with help from its cognate RING E3 ligase $\operatorname{Rad} 18$, transfers a single Ub molecule to a specific lysine (K164) on PCNA [55], a signal that promotes the switching from normal replicative polymerases to Y-family translesion polymerases to bypass a DNA lesion [56, 57].

Among the E2s that show little or no preference regarding the residues to which they transfer the $\mathrm{Ub}$ moiety, the ubiquitous Ube2D family (a.k.a UbcH5) is paramount. Family members show remarkably low rates of reaction with lysine in the absence of an E3, but are highly activated upon binding to a vast array of RING- 
type E3s. The Ube2D family is essentially indiscriminate and, once activated, will transfer Ub to any lysine residue that comes near its active site. Although this property makes Ube2D family members popular reagents for in vitro studies, it also has made identification of their biologically relevant partners and substrates challenging.

Finally, it is important to appreciate that some or all linkage-specific chain-building E2s can only perform their function on substrates that have previously been acted upon by another E2, a priming E2. This is because the highly specific chain builders such as Ube2N, Ube2S, and Ube2R1 can only transfer their conjugated $\mathrm{Ub}$ to another $\mathrm{Ub}$ molecule. This leads to a division of labor among E2s in which one E2 initiates or primes chain synthesis and a second E2 builds and extends the polyUb chain $[11,58]$. Such a strategy is used for substrates of the APC/C E3 ligase complex during cell cycle progression. Either Ube2C or a Ube2D family member transfers the first $\mathrm{Ub}$ onto human $\mathrm{APC} / \mathrm{C}$ substrates and Ube2S then builds the K11-linked polyUb chains that are a hallmark of APC/C-mediated proteasomal degradation $[59,60]$. Interestingly, the $\mathrm{APC} / \mathrm{C}$ appears to repurpose its RING subunit to bind and track the growing $\mathrm{Ub}$ chain during Ube2S-mediated catalysis, presumably inhibiting incorrect chain building by the promiscuous Ube2D E2s $[37,38]$.

In the case of $\mathrm{APC} / \mathrm{C}$, the monoubiquitylated substrate is an intermediate that most likely does not disassociate from the APC/C complex. Here different E2s work with a common E3. An interesting contrast is PCNA, whose mono- and polyubiquitylation represent separate signals that have different functional outcomes. As discussed above, PCNA is monoubiquitylated by the Ube2A/B (Rad6) E2s and the RING E3 Rad18 during postreplicative DNA damage repair [55-57]. A second E2/E3 pair, namely, Ube2N/Ube2V2 and the RING E3 Rad5, together builds a K63-linked chain at the same site, to create a signal that promotes template-switching and engagement of the homologous recombination machinery $[55,61]$. Our survey of the literature is consistent with an interpretation where Ube $2 \mathrm{~N}$ can only transfer $\mathrm{Ub}$ to the K63 of another $\mathrm{Ub}$, implying that monoubiquitylated PCNA serves as the substrate in this reaction. An unusual alternative mechanism for the modification of PCNA with K63-linked chains has recently been proposed in which K63-linked polyUb is built directly onto the active site cysteine of Ube2N. The K63-linked chain is subsequently transferred via transthiolation to another E2, Rad6, which then transfers the preformed chain en bloc to K164 on PCNA [62]. Regardless of which mechanism prevails, the process requires a division of labor between two E2s.
Our final example involves the E2 Ube2W, with its unique ability to monoubiquitylate proteins on their $\mathrm{N}$-termini. Early in vitro studies demonstrated that a substrate that is modified by Ube $2 \mathrm{~W}$ can serve as the template for chain building by Ube2N and Ube2K [11]. Several recent studies indicate that Ube $2 \mathrm{~W}$ may work as a chain-initiating E2 in the innate immune response where K63-linked chains play a critical role. The functional importance of Ube2W in the innate immune system was recently established in vivo using knockout mice [63]. Ube2W appears to monoubiquitylate the RING E3 ligases TRIM5 $\alpha$ and TRIM21, a prerequisite for their K63 polyubiquitylation by Ube2N/Ube2V2 [17, 64].

These examples demonstrate that, whether on their own or in tandem, E2s (activated by RING-type E3s) dictate the nature of the product and therefore the ultimate signal generated and its biological outcome.

\section{Regulation of E2 activity}

The central role that E2s play in Ub signaling makes them attractive targets for regulatory control. Although an understanding of E2 regulation is still emerging, it is clear that E2s can be regulated by multiple mechanisms. We have already described the most general mechanism whereby E2 reactivity is regulated via interaction with a RING-type E3 to promote a closed, more reactive E2 $\mathrm{Ub}$ conformation. Progress is being made on other aspects of E2 regulation. Here we give an overview of the regulatory mechanisms identified to date and highlight emerging themes.

Non-covalent modulation of E2 activity by backside binding

Modulation of activity through non-covalent interactions is a general mechanism of enzyme regulation. On E2s, the site used most frequently for regulatory non-covalent binding events is the so-called 'backside' surface (Table 1). Located on the face opposite from the catalytic pocket, the surface is made from UBC domain residues on the $\beta$-sheet, $\mathrm{C}$-terminal end of helix $\alpha 1$ and the following loop, and may also include the $\mathrm{C}$-terminus of helix 4 (Figure 1A). The earliest reported backside interaction was of $\mathrm{Ub}$ non-covalently bound to Ube2D3 via the hydrophobic patch centered on I44, a Ub surface used in many different protein-protein interactions [65]. Although weak in affinity $\left(\sim 300 \mu \mathrm{M} K_{\mathrm{D}}\right)$, the interaction promotes an increase in processivity of polyUb chain building by Ube2D3 [66-69]. The modulator can be free $\mathrm{Ub}$ or $\mathrm{Ub}$ that is conjugated to another Ube2D3 molecule (in a piggyback manner), which may serve to increase the local concentration of E2 Ub conjugates near an E3 
(and therefore, a substrate) [65]. Backside binding by Ub increases the intrinsic lysine reactivity of Ube2D2 Ub [70], indicating an allosteric effect, although the details of how the binding at a distal site affects active site reactivity remain to be defined experimentally.

The chain-building activity of other E2s is enhanced by Ub backside binding as well. The interaction is critical for the E3-independent ability of Ube2B to build K11-linked polyUb chains [71]. Similarly, intrinsic chain-building ability of the yeast homolog of Ube2B, Rad6, is enhanced by Ub binding [72]. Finally, the Ubl SUMO binds to the backside of its E2, Ube2I, where the interaction also has a positive effect on SUMO chain building [73]. Although the structural details of these interactions may vary, they define a general regulatory mechanism by which non-covalent binding of a $\mathrm{Ub} / \mathrm{Ubl}$ to the distal backside surface has a positive effect on an E2's chain-building efficiency.

Somewhat paradoxically, non-covalent binding of $\mathrm{Ub}$ to certain E2s negatively affects chain building and processivity. The observed preference of Ube2E3 to generate monoubiquitylated products arises from specific interactions involving $\mathrm{K} 48$ on $\mathrm{Ub}$ and backside residues of the E2 [74]. Disruption of this interaction by mutation of either $\mathrm{Ub}$ or E2 backside residues results in the rapid generation of K63-linked Ub chains by Ube2E3. Thus, this E2 can be thought of having an intrinsic ability to build polyUb chains that is inhibited by Ub binding on its backside. The two opposite effects of backside Ub binding suggest that it can act as either a throttle or a brake for chain building.

Some RING-type E3s have accessory regions that bind to the backside surface of E2s and modulate their activity. As the examples below demonstrate, this E3 region is frequently an $\alpha$-helical segment outside the RING domain, and the binding results in either increased affinity for E2 Ub conjugates and/or allosteric affects on E2 Ub activity. Notably, the E2s that have been reported to engage in backside binding with an E3 also bind $\mathrm{Ub}$ (or Ubl) on their backside (see Table 1). This sets up a competition between the two possible non-covalent interactions. Consistent with this notion, the backside-binding element of the E3 AO7 decreases the processivity of chain building by the E2 Ube2D2 [70, 75]. Similarly, backside binding by accessory elements of the RING E3 Rad18 inhibits the intrinsic chain-forming activity of Ube2B, thus promoting monoubiquitylation of PCNA and histone 2B [71, 72].

In an interesting variation on the theme, the SUMO E3 ligase, RanBP2, binds to the backside of Ube2I. Lacking a RING domain, RanBP2 contains a natively unfolded region known as internal repeat region 1 (IR1) that ex- hibits SUMO E3 ligase activity [76, 77]. IR1 appears to stabilize a closed conformation of the Ube2I $\sim$ SUMO1 conjugate via contacts on the canonical E3-binding surface of Ube2I, the backside of Ube2I, and by interactions with SUMO [78]. Precisely how IR1 enhances the transfer of SUMO remains mechanistically unclear [78].

Multi-subunit E3s also make use of the E2 backside surface. The APC/C engages Ube2C using both a RING subunit Apc11 and the WHB domain of a cullin subunit Apc2, the latter interaction being via the E2's backside [79]. While use of two subunits to recruit the E2 serves to ensure specificity for Ube2C, the additional interaction also appears to direct substrate ubiquitylation by reducing the degrees of freedom available for the E2/RING assembly. Cue1, a transmembrane subunit of the yeast ERAD RING E3 complex, has an $\alpha$-helix that binds to the backside of the ERAD E2, Ubc7, a homologue of human Ube2G2. Cue1 binding tethers Ubc7 to the membrane and increases the affinity of Ubc7 for its ERAD E3 $[80,81]$. But in addition to localizing the E2, Cue1 binding modulates $\mathrm{Ubc} 7$ activity by enhancing $\mathrm{Ub}$ loading of the E2 by E1 and by allosterically opening loops around the E2 active site. Analogously, the aforementioned human ERAD E3 ligase, gp78, uses the non-RING G2BR to interact with the backside of its E2, Ube2G2 [49]. Remarkably, even G2BR binding in trans can increase the affinity of Ube2G2 Ub for the gp78 RING and can enhance both E3-dependent and E3-independent Ub transfer activity, suggesting cooperative allosteric interactions between RING and G2BR binding [49, 50]. The situation is different in the case of uncharged Ube2G2, which binds the E3 with lower affinity due to loss of some G2BR contacts, an effect that promotes dissociation of reacted (or inactive) E2 from the E3, allowing for exchange with active E2 Ub conjugate [49, 50, 82]. In sum, these reciprocal sets of allosteric interactions represent an exquisite example of how a RING and an accessory feature of an E3 ligase can work synergistically through an E2 to ensure processive rounds of ubiquitylation.

\section{Covalent modulation of E2 reactivity}

A second common mechanism of enzyme regulation is through covalent post-translational modifications that modulate activity. There are a growing number of examples of E2s that are themselves the target of ubiquitylation. The stability of yeast $\mathrm{Ubc} 7$ is dependent on the presence of its binding partner, Cue1, a subunit of the ERAD complex (see above). Under limiting Cue1 conditions, a polyUb chain is synthesized on the Ubc7 active-site cysteine that targets it for proteasomal degradation [83]. This provides a highly effective way to 
regulate E2 activity, namely, by destroying the E2. Chain building directly on E2 active sites has only been reported in limited cases (e.g., Ubc7 and its human homolog Ube2G2 [84], and Ube2D in collaboration with the bacterial effector $\mathrm{SspH} 2$ [85]). However, considering that their detection requires special conditions to inhibit the loss of thioester-linked chains during sample handling, it is still unclear how general the feature might be among E2s.

Ubiquitylation of E2s on lysine residues also occurs. Ube2T, the E2 involved in the Fanconi Anemia DNA repair pathway, transfers $\mathrm{Ub}$ to a lysine near its active site and two lysines located in its C-terminal extension [54]. Ubiquitylated Ube2T has been observed in vitro and in cells, and its production is enhanced by the E3, FANCL. However, unlike Ubc 7 autoubiquitylation, (multi)-monoubiquitylated Ube2T does not signal for its degradation, but has decreased Ub transfer activity in vitro. Ube2E1 provides a third example of E2 regulation by autoubiquitylation. Similar to Ube2T, ubiquitylation results in inhibition of E2 activity [86]. Modification occurs on a lysine near the active site and on lysines in the unstructured N-terminal extension of Ube2E1. Notably, it is ubiquitylation of Ube2E1's N-terminal extension that gives rise to the inhibition. Deletion of the N-terminal extension of Ube2E family members switches their (in vitro) activity from mono- to polyubiquitylation, although a molecular mechanism for the inhibitory function of the N-terminus has yet to be been defined [87]. In summary, the examples to date demonstrate that, like ubiquitylation of target proteins, ubiquitylation of E2s themselves can regulate cellular E2 activity either by controlling E2 protein levels or by controlling E2 activity per se. The first mechanism, degradation, is irreversible and it remains to be determined whether regulation via monoubiquitylation of E2s can be reversed by one or more of the myriad of deubiquitylating enzymes in the cell.

The Ubl SUMO can also act as a covalent modulator of E2 activity. The SUMO E2 Ube2I is itself regulated by SUMOylation, although the modification likely occurs in trans as the modified lysine, in human Ube2I on helix $\alpha 1$, is too distant from the active site. The consequence of Ube2I SUMOylation appears to be context-dependent: it can increase, decrease, or have no effect on SUMO transfer activity, depending on the location of SUMO attachment and types of E2 contacts made by the E3 or the substrate $[88,89]$. Targets that contain SUMO-interacting motifs (SIMs) are more readily recruited to SUMO-Ube2I with a concomitant increase in (E3-independent) SUMO transfer by Ube2I, but the modification appears to block binding interfaces important for some E3s and (non-SIM-containing) target substrates [89].
Thus, autoSUMOylation is a mechanism by which cells may simultaneously tune SUMOylation of multiple substrates, increasing some, decreasing some, and allowing others to remain at a steady state. A second example of SUMO modification of an E2 is the polyUb chain-building E2, Ube2K [52]. Like Ube2I, the site of modification is on helix $\alpha 1$, but on a residue in the E1 and E3 binding interface (Figure 1), consistent with the decreased activity observed in vitro.

There is growing evidence that E2s can be modulated by other covalent modifications, including those associated with oxidative stress. The E2 Ube2E3 regulates the activity of Nrf2, a transcription factor that induces expression of anti-oxidant genes to neutralize reactive oxygen species and restore redox homeostasis [90]. Alkylation of non-catalytic C136 of Ube2E3 (to mimic its oxidation) results in constitutive binding of the E2 to Nrf2, increasing its half-life and thus its transcriptional activity [90]. Although the mechanistic details of this regulation are not fully understood, this regulation also depends on the catalytic activity of Ube2E3. Intriguingly, Ube2E3's C136 replaces the proline in a conserved HPN triad (Figure 1A), which has been reported to be required for E2 activity [51]. In a second example, Ube2I is reversibly inhibited by disulfide bond formation between its catalytic cysteine and that of the SUMO1 E1 subunit Uba2 [91]. While very high levels of oxidative stress can thereby lead to global deSUMOylation, this pathway may serve highly specific functions in the context of redox signaling, e.g., upon macrophage activation [91]. Intriguingly, several chemotherapeutic drugs used in the treatment of acute myeloid leukemia induce the SUMO E1 and E2 disulfide bond formation and cause loss of SUMOylation [92]. Similarly, a disulfide bond formed between the Ub E1 Uba1 and the E2 Ube2R1 upon oxidative stress is associated with increased Ube2R1 substrate stability and delayed cell cycle progression [93]. In summary, these E2 modifications all seem to function as redox sensors that chemically alter the E2 active site and therefore its activity.

Our final example of modulation by covalent modification of E2s involves pathogenic bacteria that invade eukaryotes. By introducing effector proteins directly into the host cell, they target a variety of pathways to facilitate invasion, inhibit innate immune response, and establish an environment for replication. Not surprisingly, such pathogens have evolved a variety of ways to exploit the Ub signaling pathways, and the regulation of specific E2s is one such strategy. An effector protein from the enteric pathogen Shigella, Osp1, deamidates a glutamine residue in Ube2N, resulting in inhibition of NF- $\kappa B$ signaling and the host immune response [94]. Although Osp1 can effectively compete with E3s for Ube2N 
binding, the primary mode of inhibition by Osp 1 is the deamidation of Q100 to yield a glutamate residue [95]. This irreversible modification places a negative charge at the beginning of the Ube $2 \mathrm{~N}$ crossover helix, a structural feature known to be involved in the closed E2 Ub conformation and activation by RING-type E3s [29, 31].

Examples of the most common covalent modification to modulate protein activity, phosphorylation, are strikingly underrepresented among E2s. Early studies reported functionally important phosphorylation in E2 extensions [96-98], but there are a growing number of identified phosphosites that are within UBC domains. Of particular interest are modifications near the active site. For example, CK2-mediated phosphorylation on a crossover helix residue of yeast Cdc34 (S130) appears to repulse the acidic loop that can occlude the catalytic cleft, resulting in its opening $[99,100]$. This affects E2 charging as well as reactivity of the Cdc34 Ub thioester in a mechanism that may apply to all E2s featuring an acidic loop [100]. Furthermore, phosphorylation by Cdk-9 on a serine referred to here as the "gateway residue" (Figure 1) activates Ube2A [101-103]. The gateway residue is in a loop that forms the opening of the E2 active-site cleft and is most often an aspartate or serine followed by proline (the Cdk-9 consensus site) in human E2s. Mutation of the gateway aspartate in the Ube2D family severely reduces $\mathrm{Ub}$ transfer to lysine side chains [33]. Simulations have also suggested that E2s with a gateway aspartate (but not a glutamate) are constitutively active, whereas the activity of E2s with a gateway serine (but not a threonine) may be controlled by phosphorylation [104]. The hypothesis awaits experimental confirmation, but provides the possibility of a unifying mechanism that links E2 activity and phosphorylation near the active site.

\section{Modulation of E2 activity by transcriptional/translation- al control}

As discussed above, protein degradation signaled by polyubiquitylation is a mechanism by which the ubiquitylation machinery can control protein levels. E2 transcription and translation are also emerging as important strategies for long-term regulation of $\mathrm{Ub} / \mathrm{Ubl}$ transfer. Both intrinsic and external forces take advantage of this strategy. Ube2I expression is increased in response to $17 \beta$-estradiol under the control of the estrogen receptor $(\mathrm{ER} \alpha)$ and nuclear factor Y [105]. Ube2L3 undergoes transcriptional regulation in response to aryl hydrocarbon receptor (AHR) signaling [106]. The resulting increase in Ube2L3 levels leads to degradation of cell cycle control proteins, identifying a connection between AHR signaling and the previously established role that Ube2L3 plays in cell cycle regulation [107]. Epstein-Barr virus (EBV) targets E2s to control host cells by regulating translation of the E2 BIRC6. The latter is an inhibitor of apoptosis protein and its degradation by Nrdp1 induces apoptosis in response to apoptotic stimuli [108]. An EBV-encoded microRNA (BART15-3p) that leads to host cell apoptosis specifically targets the E2, BIRC6, leading to a decrease in translation of the BIRC6 mRNA without affecting the mRNA stability [109].

\section{Modulation of E2 activity by small molecules}

Targeting specific E2s with small molecules is becoming more feasible thanks to the large amount of structural and biochemical information available on E2s, both on their own and in active complexes. Once discovered, selective small-molecule inhibitors can serve as powerful tools to study the function and physiological roles of E2s and, possibly, as leads for drug discovery. Due to its role in the immune response, there is interest in Ube2N as a potential drug target. A known NF- $\kappa \mathrm{B}$ inhibitor (NSC697923) covalently modifies the catalytic cysteine of Ube $2 \mathrm{~N}$ and binds to a cleft in the active site that is not accessible in other E2s, thus providing specificity [110]. Structure-based mutations confirmed that NSC697923 inhibition of NF- $\mathrm{kB}$ signaling and DNA damage response in cells is due specifically to the small molecule's action on Ube2N. Another small-molecule inhibitor (CC0651) selectively inhibits Ube2R1, the specialized E2 for SCF E3 ligases, which builds K48-linked polyUb chains on its targets for proteasomal degradation. In a co-crystal structure of Ube2R1, Ub, and CC0651, the inhibitor is sandwiched between the E2 and Ub [111]. Remarkably, the resulting non-covalent complex resembles the closed, activated E2 Ub conjugate seen for other E2s (Figure 2). The inhibitor increases RING affinity for the E2, but decreases E2 Ub hydrolysis rates perhaps by acting as a molecular glue. Notably, while the CC0651-binding surfaces on both the E2 and $\mathrm{Ub}$ are rather flat and therefore not obvious candidates for small-molecule binding, the insight that the two proteins together form a targetable interface may guide future efforts to design or screen for small-molecule inhibitors specific to other E2s. Development of small-molecule inhibitors will allow researchers to tie together the idiosyncratic roles E2s play biologically with what is known mechanistically about how they are activated and regulated.

\section{Expanding the realm: E2s as direct regulators of enzyme activity}

OTUB1 is a deubiquitinase (DUB) that selectively hydrolyzes $\mathrm{K} 48$-linked polyUb chains and, therefore, can play a regulatory role in modulating $\mathrm{Ub}$ signaling. Rather 
unexpectedly, OTUB1 DUB activity is enhanced by interaction with free E2s. Binding of Ube2D2 stabilizes the disordered OTUB1 N-terminus in an $\alpha$-helical conformation, which completes the binding site for K48-linked diUb. The E2-mediated conformational change decreases the $K_{\mathrm{m}}$ of OTUB1 for diUb by over 35 -fold, thereby enhancing the rate of OTUB1-dependent polyUb degradation [2]. This is an example of an E2 acting as an effector protein to stimulate enzyme (DUB) activity. But this is not the only regulatory interaction involving E2s and OTUB1. OTUB1 can also simultaneously bind free $\mathrm{Ub}$ and certain E2 $\sim \mathrm{Ub}$ conjugates (e.g., Ube2D(1/2/3) Ub, Ube2E1 Ub, or Ube2N Ub). The two Ub moieties occupy both Ub-binding sites on OTUB1 and effectively inhibit its DUB activity. Furthermore, the E2 Ub conjugate in the OTUB1 complex is bound in a less reactive open conformation and the E3-binding surface of the E2 is occupied by OTUB1 binding. On this basis, it has been proposed that OTUB1 inhibits the ability of E2 $\mathrm{Ub}$ conjugates to participate in Ub transfer reactions and in building polyUb chains [2]. Thus, OTUB $1 / \mathrm{E} 2 \sim \mathrm{Ub} / \mathrm{Ub}$ complexes may inhibit both E2 Ub activity and DUB activity, whereas OTUB1/E2 complexes can stimulate cleavage of K48-linked chains. These findings suggest a model where OTUB1 is poised to regulate either polyUb chain elongation or degradation and the type of regulation depends on the levels of free $\mathrm{Ub}$ and the relative levels of free E2.

A final example comes from the world of pathogenic bacteria. The effector protein OspG, is found in several pathogenic strains of Shigella, Yersinia and E. coli, plays a role in tempering innate immune responses. Shigella lacking OspG induce a much stronger inflammatory response than wild-type Shigella [112]. Sequence analysis suggested that OspG is a kinase, so it was surprising when a two-hybrid screen found that OspG selectively interacts with E2 Ub conjugates [112]. Rather than serving as substrates for phosphorylation by OspG, E2 Ub conjugates regulate its kinase activity [113]. A co-crystal structure of OspG and Ube2D3 Ub revealed that the E2 $\sim \mathrm{Ub}$ conjugate binds in an open (inactive) conformation while stabilizing OspG in an active conformation with its kinase active site fully accessible to substrates [3]. Thus, although OspG by itself is a poor kinase, it uses a clever strategy whereby it recognizes and binds tightly to E2 Ub conjugates present at relatively high concentration in its host cell to stimulate its enzymatic activity.

\section{Concluding remarks}

We have endeavored here to define the current state of understanding for the central enzymes in the trio re- quired to attach $\mathrm{Ub}$ to proteins. Three decades after their initial discovery and characterization $[6,114]$, there are finally answers to the questions posed earlier in this review, namely, "What keeps the reactivity of an E2 Ub low?" and "How is reactivity stimulated by E3s?". There is a growing understanding of the ways in which E2s can limit themselves to monoubiquitylating substrates or building chains efficiently and with fidelity. But we still lack fundamental information such as the intrinsic reactivity (and therefore side chain specificity) of some human E2s, and the identification of biological E2/E3/ substrate sets remains an enormous experimental hurdle. A combination of biochemical approaches and use of emerging gene-editing approaches will allow progress on these and other fronts in the near future.

It is not surprising that E2s are regulated by other components of the ubiquitylation machinery, although the diversity of strategies utilized is unexpected. Less predictable is the ability of E2s to serve as regulators of other proteins and enzymes. Although the number of defined examples is still small, we expect this to be an emerging theme. In this regard, it is worth noting that a large-scale two-hybrid screen involving over 40 human E2 and E2-like proteins identified $>200$ unique interacting proteins [115]. Of these, $30 \%$ are E3 ligases and another $\sim 10 \%$ are proteins involved in the Ub transfer pathways or deubiquitylation. But what of the remaining $60 \%$ of identified proteins? We expect that the list contains numerous additional examples where the small and versatile UBC domain functions outside of canonical E1/ $\mathrm{E} 2 / \mathrm{E} 3 \mathrm{Ub}$ transfer pathways, likely in a regulatory capacity. Apparently, there is still much we do not know about E2s.

\section{Acknowledgments}

This work was supported by NIH grants R01 GM088055 (to REK) and R01 GM098503 (to PSB). MS is supported by NIH 2T32 CA080416.

\section{References}

1 McGinty RK, Henrici RC, Tan S. Crystal structure of the PRC1 ubiquitylation module bound to the nucleosome. $\mathrm{Na}$ ture 2014; 514:591-596.

2 Wiener R, DiBello AT, Lombardi PM, et al. E2 ubiquitin-conjugating enzymes regulate the deubiquitinating activity of OTUB1. Nat Struct Mol Biol 2013; 20:1033-1039.

3 Pruneda JN, Smith FD, Daurie A, et al. E2 Ub conjugates regulate the kinase activity of Shigella effector OspG during pathogenesis. EMBO J 2014; 33:437-449.

4 Schelpe J, Monté D, Dewitte F, Sixma TK, Rucktooa P. Structure of UBE2Z provides functional insight into specificity in the FAT10 conjugation machinery. J Biol Chem 2016; 
291:630-639.

5 Siepmann TJ. Protein interactions within the N-end rule ubiquitin ligation pathway. J Biol Chem 2003; 278:9448-9457.

6 Pickart CM, Rose IA. Functional heterogeneity of ubiquitin carrier proteins. J Biol Chem 1985; 260:1573-1581.

7 Metzger MB, Pruneda JN, Klevit RE, Weissman AM. RINGtype E3 ligases: Master manipulators of E2 ubiquitin-conjugating enzymes and ubiquitination. Biochim Biophys Acta 2014; 1843:47-60.

8 Williams C, van den Berg M, Sprenger RR, Distel B. A conserved cysteine is essential for Pex4p-dependent ubiquitination of the peroxisomal import receptor Pex5p. J Biol Chem 2007; 282:22534-22543.

9 Léon S, Subramani S. A conserved cysteine residue of pichia pastoris Pex20p is essential for its recycling from the peroxisome to the cytosol. J Biol Chem 2007; 282:7424-7430.

10 Wenzel DM, Lissounov A, Brzovic PS, Klevit RE. UBCH7 reactivity profile reveals parkin and HHARI to be RING/ HECT hybrids. Nature 2011; 474:105-108.

11 Christensen DE, Brzovic PS, Klevit RE. E2-BRCA1 RING interactions dictate synthesis of mono- or specific polyubiquitin chain linkages. Nat Struct Mol Biol 2007; 14:941-948.

12 Zhang Y, Zhou X, Zhao L, et al. UBE2W interacts with FAN$\mathrm{CL}$ and regulates the monoubiquitination of Fanconi anemia protein FANCD2. Mol Cells 2011; 31:113-122.

13 Vittal V, Shi L, Wenzel DM, et al. Intrinsic disorder drives N-terminal ubiquitination by Ube2w. Nat Chem Biol 2015; 11:83-89.

14 Tatham MH, Plechanovova A, Jaffray EG, Salmen H, Hay RT. Ube2W conjugates ubiquitin to alpha-amino groups of protein N-termini. Biochem J 2013; 453:137-145.

15 Scaglione KM, Basrur V, Ashraf NS, et al. The ubiquitin-conjugating enzyme (E2) Ube2w ubiquitinates the N-terminus of substrates. J Biol Chem 2013; 288:18784-18788.

16 Qi C, Li DF, Feng L, et al. Biochemical and structural characterization of a novel ubiquitin-conjugating enzyme E2 from Agrocybe aegeria reveals Ube2w family-specific properties. Sci Rep 2015; 5:16056.

17 Fletcher AJ, Christensen DE, Nelson C, et al. TRIM5 $\alpha$ requires Ube2W to anchor Lys63-linked ubiquitin chains and restrict reverse transcription. EMBO J 2015; 34:2078-2095.

18 Geng J, Klionsky DJ. The Atg8 and Atg12 ubiquitin-like conjugation systems in macroautophagy. Protein modifications: beyond the usual suspects; review series. EMBO Rep 2008; 9:859-864.

19 Slobodkin MR, Elazar Z. The Atg8 family: multifunctional ubiquitin-like key regulators of autophagy. Essays Biochem 2013; 55:51-64.

20 McDowell GS, Philpott A. Non-canonical ubiquitylation: mechanisms and consequences. Int J Biochem Cell Biol 2013; 45:1833-1842.

21 Wang X, Herr RA, Chua WJ, Lybarger L, Wiertz EJ, Hansen TH. Ubiquitination of serine, threonine, or lysine residues on the cytoplasmic tail can induce ERAD of MHC-I by viral E3 ligase mK3. J Cell Biol 2007; 177:613-624.

22 Wang X, Herr RA, Rabelink M, Hoeben RC, Wiertz EJ, Hansen TH. Ube2j2 ubiquitinates hydroxylated amino acids on ER-associated degradation substrates. J Cell Biol 2009; 187:655-668.
23 Boban M, Ljungdahl PO, Foisner R. Atypical ubiquitylation in yeast targets lysine-less Asi2 for proteasomal degradation. J Biol Chem 2015; 290:2489-2495.

24 Zheng N, Wang P, Jeffrey PD, Pavletich NP. Structure of a c-Cbl-UbcH7 complex: RING domain function in ubiquitin-protein ligases. Cell 2000; 102:533-539.

25 Brzovic PS, Keeffe JR, Nishikawa $\mathrm{H}$, et al. Binding and recognition in the assembly of an active BRCA1/BARD1 ubiquitin-ligase complex. Proc Natl Acad Sci USA 2003; 100:56465651.

26 Huang L, Kinnucan E, Wang G, et al. Structure of an E6AP$\mathrm{UbcH7}$ complex: insights into ubiquitination by the E2-E3 enzyme cascade. Science 1999; 286:1321-1326.

27 Eletr ZM, Huang DT, Duda DM, Schulman BA, Kuhlman B. E2 conjugating enzymes must disengage from their E1 enzymes before E3-dependent ubiquitin and ubiquitin-like transfer. Nat Struct Mol Biol 2005; 12:933-934.

28 Sheng Y, Hong JH, Doherty R, et al. A human ubiquitin conjugating enzyme (E2)-HECT E3 ligase structure-function screen. Mol Cell Proteomics 2012; 11:329-341.

29 Pruneda JN, Stoll KE, Bolton LJ, Brzovic PS, Klevit RE. Ubiquitin in motion: structural studies of the ubiquitin-conjugating enzyme-ubiquitin conjugate. Biochemistry 2011; 50:1624-1633.

30 Soss SE, Klevit RE, Chazin WJ. Activation of UbcH5c $\sim \mathrm{Ub}$ is the result of a shift in interdomain motions of the conjugate bound to U-box E3 ligase E4B. Biochemistry 2013; 52:29912999.

31 Pruneda JN, Littlefield PJ, Soss SE, et al. Structure of an E3:E2 Ub complex reveals an allosteric mechanism shared among RING/U-box ligases. Mol Cell 2012; 47:933-942.

32 Dou H, Buetow L, Sibbet GJ, Cameron K, Huang DT. BIRC7-E2 ubiquitin conjugate structure reveals the mechanism of ubiquitin transfer by a RING dimer. Nat Struct Mol Biol 2012; 19:876-883.

33 Plechanovová A, Jaffray EG, Tatham MH, Naismith JH, Hay RT. Structure of a RING E3 ligase and ubiquitin-loaded E2 primed for catalysis. Nature 2012; 489:115-120.

34 Branigan E, Plechanovova A, Jaffray EG, Naismith JH, Hay RT. Structural basis for the RING-catalyzed synthesis of K63linked ubiquitin chains. Nat Struct Mol Biol 2015; 22:597602.

35 Hamilton KS, Ellison MJ, Barber KR, et al. Structure of a conjugating enzyme-ubiquitin thiolester intermediate reveals a novel role for the ubiquitin tail. Structure 2001; 9:897-904.

36 Wickliffe KE, Lorenz S, Wemmer DE, Kuriyan J, Rape M. The mechanism of linkage-specific ubiquitin chain elongation by a single-subunit E2. Cell 2011; 144:769-781.

37 Brown NG, Watson ER, Weissmann F, et al. Mechanism of polyubiquitination by human anaphase-promoting complex: RING repurposing for ubiquitin chain assembly. Mol Cell 2014; 56:246-260.

38 Kelly A, Wickliffe KE, Song L, Fedrigo I, Rape M. Ubiquitin chain elongation requires E3-dependent tracking of the emerging conjugate. Mol Cell 2014; 56:232-245.

39 Kamadurai HB, Souphron J, Scott DC, et al. Insights into ubiquitin transfer cascades from a structure of a $\mathrm{UbcH5B}$ approximately ubiquitin-HECT(NEDD4L) complex. Mol Cell 2009; 36:1095-1102. 
40 Lechtenberg BC, Rajput A, Sanishvili R, et al. Structure of a HOIP/E2 ubiquitin complex reveals RBR E3 ligase mechanism and regulation. Nature 2016; 529:546-550.

41 Eddins MJ, Carlile CM, Gomez KM, Pickart CM, Wolberger C. Mms2-Ubc13 covalently bound to ubiquitin reveals the structural basis of linkage-specific polyubiquitin chain formation. Nat Struct Mol Biol 2006; 13:915-920.

42 Rodrigo-Brenni MC, Foster SA, Morgan DO. Catalysis of lysine 48-specific ubiquitin chain assembly by residues in E2 and ubiquitin. Mol Cell 2010; 39:548-559.

43 Middleton AJ, Day CL. The molecular basis of lysine 48 ubiquitin chain synthesis by Ube2K. Sci Rep 2015; 5:16793.

44 Kleiger G, Saha A, Lewis S, Kuhlman B, Deshaies RJ. Rapid E2-E3 assembly and disassembly enable processive ubiquitylation of cullin-RING ubiquitin ligase substrates. Cell 2009; 139:957-968.

45 Ziemba A, Hill S, Sandoval D, Webb K, Bennett EJ, Kleiger G. Multimodal mechanism of action for the Cde34 acidic loop: a case study for why ubiquitin-conjugating enzymes have loops and tails. J Biol Chem 2013; 288:34882-34896.

46 Chong RA, Wu K, Spratt DE, et al. Pivotal role for the ubiquitin Y59-E51 loop in lysine 48 polyubiquitination. Proc Natl Acad Sci USA 2014; 111:8434-8439.

47 Kleiger G, Hao B, Mohl DA, Deshaies RJ. The acidic tail of the $\mathrm{Cdc} 34$ ubiquitin-conjugating enzyme functions in both binding to and catalysis with ubiquitin ligase SCFCdc4. J Biol Chem 2009; 284:36012-36023.

48 Sandoval D, Hill S, Ziemba A, Lewis S, Kuhlman B, Kleiger G. Ubiquitin-conjugating enzyme Cdc34 and ubiquitin ligase Skp1-cullin-F-box ligase (SCF) interact through multiple conformations. J Biol Chem 2015; 290:1106-1118.

49 Das R, Liang YH, Mariano J, et al. Allosteric regulation of $\mathrm{E} 2$ :E3 interactions promote a processive ubiquitination machine. EMBO J 2013; 32:2504-2516.

50 Das R, Mariano J, Tsai YC, et al. Allosteric activation of E2RING finger-mediated ubiquitylation by a structurally defined specific E2-binding region of gp78. Mol Cell 2009; 34:674685.

51 Bernier-Villamor V, Sampson DA, Matunis MJ, Lima CD. Structural basis for E2-mediated SUMO conjugation revealed by a complex between ubiquitin-conjugating enzyme Ubc9 and RanGAP1. Cell 2002; 108:345-356.

52 Pichler A, Knipscheer P, Oberhofer E, et al. SUMO modification of the ubiquitin-conjugating enzyme E2-25K. Nat Struct Mol Biol 2005; 12:264-269.

53 Yunus AA, Lima CD. Lysine activation and functional analysis of E2-mediated conjugation in the SUMO pathway. Nat Struct Mol Biol 2006; 13:491-499.

54 Machida YJ, Machida Y, Chen Y, et al. UBE2T is the E2 in the fanconi anemia pathway and undergoes negative autoregulation. Mol Cell 2006; 23:589-596.

55 Hoege C, Pfander B, Moldovan GL, Pyrowolakis G, Jentsch S. RAD6-dependent DNA repair is linked to modification of PCNA by ubiquitin and SUMO. Nature 2002; 419:135-141.

56 Parker JL, Bielen AB, Dikic I, Ulrich HD. Contributions of ubiquitin- and PCNA-binding domains to the activity of Polymerase eta in Saccharomyces cerevisiae. Nucleic Acids Res 2007; 35:881-889.

57 Stelter P, Ulrich HD. Control of spontaneous and damage-in- duced mutagenesis by SUMO and ubiquitin conjugation. $\mathrm{Na}$ ture 2003; 425:188-191.

58 Windheim M, Peggie M, Cohen P. Two different classes of E2 ubiquitin-conjugating enzymes are required for the mono-ubiquitination of proteins and elongation by polyubiquitin chains with a specific topology. Biochem J 2008; 409:723729.

59 Williamson A, Wickliffe KE, Mellone BG, Song L, Karpen GH, Rape M. Identification of a physiological E2 module for the human anaphase-promoting complex. Proc Natl Acad Sci USA 2009; 106:18213-18218.

$60 \mathrm{Wu}$ T, Merbl Y, Huo Y, Gallop JL, Tzur A, Kirschner MW. UBE2S drives elongation of K11-linked ubiquitin chains by the anaphase-promoting complex. Proc Natl Acad Sci USA 2010; 107:1355-1360.

61 Ulrich HD, Jentsch S. Two RING finger proteins mediate cooperation between ubiquitin-conjugating enzymes in DNA repair. EMBO J 2000; 19:3388-3397.

62 Masuda Y, Suzuki M, Kawai H, et al. En bloc transfer of polyubiquitin chains to PCNA in vitro is mediated by two different human E2-E3 pairs. Nucleic Acids Res 2012; 40:1039410407.

63 Wang B, Merillat SA, Vincent M, et al. Loss of the ubiquitin-conjugating enzyme Ube $2 \mathrm{~W}$ results in susceptibility to early postnatal lethality and defects in skin, immune and male reproductive systems. J Biol Chem 2016; 291:3030-3042.

64 Fletcher AJ, Mallery DL, Watkinson RE, Dickson CF, James LC. Sequential ubiquitination and deubiquitination enzymes synchronize the dual sensor and effector functions of TRIM21. Proc Natl Acad Sci USA 2015; 112:10014-10019.

65 Brzovic PS, Lissounov A, Christensen DE, Hoyt DW, Klevit RE. A UbcH5/ubiquitin noncovalent complex is required for processive BRCA1-directed ubiquitination. Mol Cell 2006; 21:873-880.

66 Brzovic PS, Klevit RE. Ubiquitin transfer from the E2 perspective: why is UbcH5 so promiscuous? Cell Cycle 2006; 5:2867-2873.

67 Ranaweera RS, Yang X. Auto-ubiquitination of Mdm2 enhances its substrate ubiquitin ligase activity. J Biol Chem 2013; 288:18939-18946.

68 Page RC, Pruneda JN, Amick J, Klevit RE, Misra S. Structural insights into the conformation and oligomerization of E2 ubiquitin conjugates. Biochemistry 2012; 51:4175-4187.

69 Sakata E, Satoh T, Yamamoto S, et al. Crystal structure of Ub$\mathrm{cH} 5 \mathrm{~b} \sim$ ubiquitin intermediate: insight into the formation of the self-assembled E2 Ub conjugates. Structure 2010; 18:138147.

70 Buetow L, Gabrielsen M, Anthony NG, et al. Activation of a primed RING E3-E2-ubiquitin complex by non-covalent ubiquitin. Mol Cell 2015; 58:297-310.

71 Hibbert RG, Huang A, Boelens R, Sixma TK. E3 ligase Rad18 promotes monoubiquitination rather than ubiquitin chain formation by E2 enzyme Rad6. Proc Natl Acad Sci USA 2011; 108:5590-5595.

72 Kumar P, Magala P, Geiger-Schuller KR, Majumdar A, Tolman JR, Wolberger C. Role of a non-canonical surface of Rad6 in ubiquitin conjugating activity. Nucleic Acids Res 2015; 43:9039-9050.

73 Knipscheer P, van Dijk WJ, Olsen JV, Mann M, Sixma TK. 
Noncovalent interaction between Ubc9 and SUMO promotes SUMO chain formation. EMBO J 2007; 26:2797-2807.

74 Nguyen L, Plafker KS, Starnes A, Cook M, Klevit RE, Plafker $\mathrm{SM}$. The ubiquitin-conjugating enzyme, UbcM2, is restricted to monoubiquitylation by a two-fold mechanism that involves backside residues of E2 and Lys48 of ubiquitin. Biochemistry 2014; 53:4004-4014.

75 Li S, Liang Y-H, Mariano J, et al. Insights into ubiquitination from the unique clamp-like binding of the RING E3 AO7 to the E2 UbcH5B. J Biol Chem 2015; 290:30225-30239.

76 Pichler A, Melchior F. Ubiquitin-related modifier SUMO1 and nucleocytoplasmic transport. Traffic 2002; 3:381-387.

77 Pichler A, Knipscheer P, Saitoh H, Sixma TK, Melchior F. The RanBP2 SUMO E3 ligase is neither HECT- nor RINGtype. Nat Struct Mol Biol 2004; 11:984-991.

78 Reverter D, Lima CD. Insights into E3 ligase activity revealed by a SUMO-RanGAP1-Ubc9-Nup358 complex. Nature 2005; 435:687-692.

79 Brown NG, VanderLinden R, Watson ER, et al. RING E3 mechanism for ubiquitin ligation to a disordered substrate visualized for human anaphase-promoting complex. Proc Natl Acad Sci USA 2015; 112:5272-5279.

80 Bazirgan OA, Hampton RY. Cuelp is an activator of Ubc7p E2 activity in vitro and in vivo. J Biol Chem 2008; 283:1279712810.

81 Metzger MB, Liang YH, Das R, et al. A structurally unique E2-binding domain activates ubiquitination by the ERAD E2, Ubc7p, through multiple mechanisms. Mol Cell 2013; 50:516527.

82 Olsen SK, Lima CD. Structure of a ubiquitin E1-E2 complex: insights to E1-E2 thioester transfer. Mol Cell 2013; 49:884896.

83 Ravid T, Hochstrasser M. Autoregulation of an E2 enzyme by ubiquitin-chain assembly on its catalytic residue. Nat Cell Biol 2007; 9:422-427.

84 Li W, Tu D, Brunger AT, Ye Y. A ubiquitin ligase transfers preformed polyubiquitin chains from a conjugating enzyme to a substrate. Nature 2007; 446:333-337.

85 Levin I, Eakin C, Blanc MP, Klevit RE, Miller SI, Brzovic PS. Identification of an unconventional E3 binding surface on the $\mathrm{UbcH} 5 \sim \mathrm{Ub}$ conjugate recognized by a pathogenic bacterial E3 ligase. Proc Natl Acad Sci USA 2010; 107:2848-2853.

86 Banka PA, Behera AP, Sarkar S, Datta AB. RING E3-catalyzed E2 self-ubiquitination attenuates the activity of Ube2E ubiquitin-conjugating enzymes. J Mol Biol 2015; 427:22902304.

87 Schumacher FR, Wilson G, Day CL. The N-terminal extension of UBE2E ubiquitin-conjugating enzymes limits chain assembly. J Mol Biol 2013; 425:4099-4111.

88 Ho CW, Chen HT, Hwang J. UBC9 Autosumoylation negatively regulates sumoylation of septins in Saccharomyces cerevisiae. J Biol Chem 2011; 286:21826-21834.

89 Knipscheer P, Flotho A, Klug H, et al. Ubc9 sumoylation regulates SUMO target discrimination. Mol Cell 2008; 31:371382.

90 Plafker KS, Nguyen L, Barneche M, Mirza S, Crawford D, Plafker SM. The ubiquitin-conjugating enzyme UbcM2 can regulate the stability and activity of the antioxidant transcription factor Nrf2. J Biol Chem 2010; 285:23064-23074.
91 Bossis G, Melchior F. Regulation of SUMOylation by reversible oxidation of SUMO conjugating enzymes. Mol Cell 2006; 21:349-357.

92 Bossis G, Sarry JE, Kifagi C, et al. The ROS/SUMO axis contributes to the response of acute myeloid leukemia cells to chemotherapeutic drugs. Cell Rep 2014; 7:1815-1823.

93 Doris KS, Rumsby EL, Morgan BA. Oxidative stress responses involve oxidation of a conserved ubiquitin pathway enzyme. Mol Cell Biol 2012; 32:4472-4481.

94 Sanada T, Kim M, Mimuro H, et al. The Shigella flexneri effector OspI deamidates UBC13 to dampen the inflammatory response. Nature 2012; 483:623-626.

95 Fu P, Zhang X, Jin M, et al. Complex structure of OspI and Ubc13: the molecular basis of Ubc13 deamidation and convergence of bacterial and host E2 recognition. PLoS Pathog 2013; 9:e1003322.

96 Block K, Boyer TG, Yew PR. Phosphorylation of the human ubiquitin-conjugating enzyme, $\mathrm{CDC} 34$, by casein kinase $2 . J$ Biol Chem 2001; 276:41049-41058.

97 Semplici F, Meggio F, Pinna LA, Oliviero S. CK2-dependent phosphorylation of the E2 ubiquitin conjugating enzyme UBC3B induces its interaction with beta- $\mathrm{TrCP}$ and enhances beta-catenin degradation. Oncogene 2002; 21:3978-3987.

98 Oh RS, Bai X, Rommens JM. Human homologs of Ubc6p ubiquitin-conjugating enzyme and phosphorylation of HsUbc6e in response to endoplasmic reticulum stress. $J$ Biol Chem 2006; 281:21480-21490.

99 Coccetti P, Tripodi F, Tedeschi G, et al. The CK2 phosphorylation of catalytic domain of $\mathrm{Cdc} 34$ modulates its activity at the G1 to $\mathrm{S}$ transition in Saccharomyces cerevisiae. Cell Cycle 2008; 7:1391-1401.

100 Papaleo E, Ranzani V, Tripodi F, et al. An acidic loop and cognate phosphorylation sites define a molecular switch that modulates ubiquitin charging activity in Cdc34-like enzymes. PLoS Comput Biol 2011; 7:e1002056.

101 Sarcevic B, Mawson A, Baker RT, Sutherland RL. Regulation of the ubiquitin-conjugating enzyme hHR6A by CDK-mediated phosphorylation. EMBO J 2002; 21:2009-2018.

102 Wood A, Schneider J, Dover J, Johnston M, Shilatifard A. The Bur1/Bur2 complex is required for histone H2B monoubiquitination by Rad6/Bre1 and histone methylation by COMPASS. Mol Cell 2005; 20:589-599.

103 Shchebet A, Karpiuk O, Kremmer E, Eick D, Johnsen SA. Phosphorylation by cyclin-dependent kinase- 9 controls ubiquitin-conjugating enzyme-2A function. Cell Cycle 2012; 11:2122-2127.

104 Valimberti I, Tiberti M, Lambrughi M, Sarcevic B, Papaleo E. E2 superfamily of ubiquitin-conjugating enzymes: constitutively active or activated through phosphorylation in the catalytic cleft. Sci Rep 2015; 5:14849.

105 Ying S, Dunnebier T, Si J, Hamann U. Estrogen receptor alpha and nuclear factor $\mathrm{Y}$ coordinately regulate the transcription of the SUMO-conjugating UBC9 gene in MCF-7 breast cancer cells. PLoS One 2013; 8:e75695.

106 Mejia-Garcia A, Gonzalez-Barbosa E, Martinez-Guzman C, et al. Activation of AHR mediates the ubiquitination and proteasome degradation of c-Fos through the induction of Ubcm4 gene expression. Toxicology 2015; 337:47-57.

107 Whitcomb EA, Dudek EJ, Liu Q, Taylor A. Novel control of 
S phase of the cell cycle by ubiquitin-conjugating enzyme $\mathrm{H} 7$. Mol Biol Cell 2009; 20:1-9.

108 Qiu XB, Markant SL, Yuan J, Goldberg AL. Nrdp1-mediated degradation of the gigantic IAP, BRUCE, is a novel pathway for triggering apoptosis. EMBO J 2004; 23:800-810.

109 Choi H, Lee H, Kim SR, Gho YS, Lee SK. Epstein-Barr virus-encoded microRNA BART15-3p promotes cell apoptosis partially by targeting BRUCE. J Virol 2013; 87:8135-8144.

110 Hodge CD, Edwards RA, Markin CJ, et al. Covalent inhibition of Ubc13 affects ubiquitin signaling and reveals active site elements important for targeting. ACS Chem Biol 2015; 10:1718-1728.

111 Huang H, Ceccarelli DF, Orlicky S, et al. E2 enzyme inhibition by stabilization of a low-affinity interface with ubiquitin. Nat Chem Biol 2014; 10:156-163.

112 Kim DW, Lenzen G, Page AL, Legrain P, Sansonetti PJ, Parsot C. The Shigella flexneri effector OspG interferes with innate immune responses by targeting ubiquitin-conjugating enzymes. Proc Natl Acad Sci USA 2005; 102:14046-14051.

113 Zhou Y, Dong N, Hu L, Shao F. The Shigella type three secretion system effector OspG directly and specifically binds to host ubiquitin for activation. PLoS One 2013; 8:e57558.

114 Hershko A, Heller H, Elias S, Ciechanover A. Components of ubiquitin-protein ligase system. Resolution, affinity purification, and role in protein breakdown. J Biol Chem 1983; 258:8206-8214.

115 Markson G, Kiel C, Hyde R, et al. Analysis of the human E2 ubiquitin conjugating enzyme protein interaction network. Genome Res 2009; 19:1905-1911.

116 Miura T, Klaus W, Gsell B, Miyamoto C, Senn H. Characterization of the binding interface between ubiquitin and class I human ubiquitin-conjugating enzyme $2 \mathrm{~b}$ by multidimensional heteronuclear NMR spectroscopy in solution. $J$ Mol Biol 1999; 290:213-228.

117 Turco E, Gallego LD, Schneider M, Köhler A. Monoubiquitination of histone $\mathrm{H} 2 \mathrm{~B}$ is intrinsic to the Bre 1 RING domain-Rad6 interaction and augmented by a second Rad6-binding site on Bre1. J Biol Chem 2015; 290:52985310.

118 Jentsch S, McGrath JP, Varshavsky A. The yeast DNA repair gene RAD6 encodes a ubiquitin-conjugating enzyme. Nature 1987; 329:131-134.

119 Summers MK, Pan B, Mukhyala K, Jackson PK. The unique N-terminus of the UbcH10 E2 enzyme controls the threshold for APC activation and enhances checkpoint regulation of the APC. Mol Cell 2008; 31:544-556.

120 Bosanac I, Phu L, Pan B, et al. Modulation of K11-linkage formation by variable loop residues within UbcH5A. $J$ Mol Biol 2011; 408:420-431.

121 Choi YS, Lee YJ, Lee SY, et al. Differential ubiquitin binding by the acidic loops of Ube2g1 and Ube2r1 enzymes distinguishes their Lys-48-ubiquitylation activities. J Biol Chem 2015; 290:2251-2263.

122 Ryu K-S, Choi Y-S, Ko J, et al. Direct characterization of E2-dependent target specificity and processivity using an artificial p27-linker-E2 ubiquitination system. BMB Rep 2008; 41:852-857.

123 Bocik WE, Sircar A, Gray JJ, Tolman JR. Mechanism of polyubiquitin chain recognition by the human ubiquitin conjugat- ing enzyme Ube2g2. J Biol Chem 2011; 286:3981-3991.

$124 \mathrm{Li} \mathrm{W}, \mathrm{Tu} \mathrm{D}, \mathrm{Li} \mathrm{L}$, et al. Mechanistic insights into active site-associated polyubiquitination by the ubiquitin-conjugating enzyme Ube2g2. Proc Natl Acad Sci USA 2009; 106:3722-3727.

125 Liu W, Shang Y, Zeng Y, et al. Dimeric Ube2g2 simultaneously engages donor and acceptor ubiquitins to form Lys48linked ubiquitin chains. EMBO J 2014; 33:46-61.

126 Kostova Z, Tsai YC, Weissman AM. Ubiquitin ligases, critical mediators of endoplasmic reticulum-associated degradation. Semin Cell Dev Biol 2007; 18:770-779.

127 Lenk U, Yu H, Walter J, et al. A role for mammalian Ubc6 homologues in ER-associated protein degradation. J Cell Sci 2002; 115:3007-3014.

128 Haldeman MT, Xia G, Kasperek EM, Pickart CM. Structure and function of ubiquitin conjugating enzyme E2-25K: the tail is a core-dependent activity element. Biochemistry 1997; 36:10526-10537.

129 Hofmann RM, Pickart CM. Noncanonical MMS2-encoded ubiquitin-conjugating enzyme functions in assembly of novel polyubiquitin chains for DNA repair. Cell 1999; 96:645-653.

130 Klemperer NS, Berleth ES, Pickart CM. A novel, arsenite-sensitive E2 of the ubiquitin pathway: purification and properties. Biochemistry 1989; 28:6035-6041.

131 Mashtalir N, Daou S, Barbour H, et al. Autodeubiquitination protects the tumor suppressor BAP1 from cytoplasmic sequestration mediated by the atypical ubiquitin ligase UBE2O. Mol Cell 2014; 54:392-406.

132 Berleth ES, Pickart CM. Mechanism of ubiquitin conjugating enzyme E2-230K: catalysis involving a thiol relay? Biochemistry 1996; 35:1664-1671.

133 Saha A, Lewis S, Kleiger G, Kuhlman B, Deshaies RJ. Essential role for ubiquitin-ubiquitin-conjugating enzyme interaction in ubiquitin discharge from $\mathrm{Cdc} 34$ to substrate. Mol Cell 2011; 42:75-83.

134 Petroski MD, Deshaies RJ. Function and regulation of cullin-RING ubiquitin ligases. Nat Rev Mol Cell Biol 2005; 6:920.

135 Lewis MJ, Saltibus LF, Hau DD, Xiao W, Spyracopoulos L. Structural basis for non-covalent interaction between ubiquitin and the ubiquitin conjugating enzyme variant human MMS2. J Biomol NMR 2006; 34:89-100.

136 Bartke T, Pohl C, Pyrowolakis G, Jentsch S. Dual role of BRUCE as an antiapoptotic IAP and a chimeric E2/E3 ubiquitin ligase. Mol Cell 2004; 14:801-811.

137 Huang DT, Ayrault O, Hunt HW, et al. E2-RING expansion of the NEDD8 cascade confers specificity to cullin modification. Mol Cell 2009; 33:483-495.

138 Lee GW, Melchior F, Matunis MJ, Mahajan R, Tian Q, Anderson P. Modification of Ran GTPase-activating protein by the small ubiquitin-related modifier SUMO-1 requires Ubc9, an E2-type ubiquitin-conjugating enzyme homologue. $J$ Biol Chem 1998; 273:6503-6507.

139 Serniwka SA, Shaw GS. The structure of the UbcH8-ubiquitin complex shows a unique ubiquitin interaction site. Biochemistry 2009; 48:12169-12179.

140 Zhao C, Beaudenon SL, Kelley ML, et al. The UbcH8 ubiquitin E2 enzyme is also the E2 enzyme for ISG15, an IFN-alpha/beta-induced ubiquitin-like protein. Proc Natl Acad Sci 
USA 2004; 101:7578-7582.

141 Scott DC, Sviderskiy VO, Monda JK, et al. Structure of a RING E3 trapped in action reveals ligation mechanism for the ubiquitin-like protein NEDD8. Cell 2014; 157:1671-1684.

142 Aichem A, Pelzer C, Lukasiak S, et al. USE1 is a bispecific conjugating enzyme for ubiquitin and FAT10, which FAT10ylates itself in cis. Nat Commun 2010; 1:13.

143 Shintani T, Mizushima N, Ogawa Y, Matsuura A, Noda T, Ohsumi Y. Apg10p, a novel protein-conjugating enzyme essential for autophagy in yeast. EMBO J 1999; 18:5234-5241.

144 Metlagel Z, Otomo C, Takaesu G, Otomo T. Structural basis of ATG3 recognition by the autophagic ubiquitin-like protein
ATG12. Proc Natl Acad Sci USA 2013; 110:18844-18849. 145 Ichimura Y, Kirisako T, Takao T, et al. A ubiquitin-like system mediates protein lipidation. Nature 2000; 408:488-492.

c) (i) $(-)$ This work is licensed under a Creative Commons Attribution-NonCommercial-NoDerivs 4.0 Unported License. The images or other third party material in this article are included in the article's Creative Commons license, unless indicated otherwise in the credit line; if the material is not included under the Creative Commons license, users will need to obtain permission from the license holder to reproduce the material. To view a copy of this license, visit http://creativecommons.org/licenses/by-nc-nd/4.0/ 